Louisiana State University

LSU Digital Commons

Faculty Publications

Department of Chemistry

$11-11-2011$

\title{
Competencies: A cure for pre-med curriculum
}

\author{
Winston A. Anderson \\ Howard University \\ Richard M. Amasino \\ University of Wisconsin-Madison \\ Manuel Ares \\ University of California, Santa Cruz \\ Utpal Banerjee \\ University of California, Los Angeles \\ Bonnie Bartel \\ Rice University
}

See next page for additional authors

Follow this and additional works at: https://digitalcommons.Isu.edu/chemistry_pubs

\section{Recommended Citation}

Anderson, W., Amasino, R., Ares, M., Banerjee, U., Bartel, B., Corces, V., Drennan, C., Elgin, S., Epstein, I., Fanning, E., Guillette, L., Handelsman, J., Hatfull, G., Hoy, R., Kelley, D., Leinwand, L., Losick, R., Lu, Y., Lynn, D., Neuhauser, C., O'Dowd, D., Olivera, T., Pevzner, P., Richards-Kortum, R., Rine, J., Sah, R., Strobel, S., Walker, G., Walt, D., Warner, I., Wessler, S., Willard, H., \& Zare, R. (2011). Competencies: A cure for pre-med curriculum. Science, 334 (6057), 760-761. https://doi.org/10.1126/science.334.6057.760-b

This Letter to the Editor is brought to you for free and open access by the Department of Chemistry at LSU Digital Commons. It has been accepted for inclusion in Faculty Publications by an authorized administrator of LSU Digital Commons. For more information, please contact ir@lsu.edu. 


\section{Authors}

Winston A. Anderson, Richard M. Amasino, Manuel Ares, Utpal Banerjee, Bonnie Bartel, Victor G. Corces, Catherine L. Drennan, Sarah C.R. Elgin, Irving R. Epstein, Ellen Fanning, Louis J. Guillette, J. O.

Handelsman, Graham F. Hatfull, Ronald Raymond Hoy, Darcy Kelley, Leslie A. Leinwand, Richard Losick, Yi Lu, David G. Lynn, Claudia Neuhauser, Diane K. O'Dowd, Toto Olivera, Pavel Pevzner, Rebecca R. Richards-

Kortum, Jasper Rine, Robert L. Sah, Scott A. Strobel, Graham C. Walker, David R. Walt, Isiah M. Warner, Sue Wessler, Huntington F. Willard, and Richard N. Zare 
Washington University in St. Louis

Washington University Open Scholarship

Biology Faculty Publications \& Presentations

Biology

$11-2011$

\section{Competencies: a cure for pre-med curriculum}

Winston Anderson

Richard Amasino

Manuel Ares

Utpal Banerjee

Bonnie Bartel

See next page for additional authors

Follow this and additional works at: https://openscholarship.wustl.edu/bio_facpubs

Part of the Biology Commons

\section{Recommended Citation}

Anderson, Winston; Amasino, Richard; Ares, Manuel; Banerjee, Utpal; Bartel, Bonnie; Corces, Victor; Drennan, Catherine; Elgin, Sarah C.R.; Epstein, Irving; Fanning, Ellen; Guillette, Louis; Handelsman, Jo; Hatfull, Graham; Hoy, Ronald; Kelley, Darcy; Leinwand, Leslie; Losick, Richard; Lu, Yi; Lynn, David; Neuhauser, Claudia; O'Dowd, Diane; Olivera, Toto; Pevzner, Pavel; Richards-Kortum, Rebecca; Rine, Jasper; Sah, Robert; Strobel, Scott; Walker, Graham; Walt, David; Warner, Isiah; Wessler, Sue; Willard, Huntington; and Zare, Richard, "Competencies: a cure for pre-med curriculum" (2011). Biology Faculty Publications \& Presentations. 184.

https://openscholarship.wustl.edu/bio_facpubs/184

This Article is brought to you for free and open access by the Biology at Washington University Open Scholarship. It has been accepted for inclusion in Biology Faculty Publications \& Presentations by an authorized administrator of Washington University Open Scholarship. For more information, please contact digital@wumail.wustl.edu. 


\section{Authors}

Winston Anderson, Richard Amasino, Manuel Ares, Utpal Banerjee, Bonnie Bartel, Victor Corces, Catherine Drennan, Sarah C.R. Elgin, Irving Epstein, Ellen Fanning, Louis Guillette, Jo Handelsman, Graham Hatfull, Ronald Hoy, Darcy Kelley, Leslie Leinwand, Richard Losick, Yi Lu, David Lynn, Claudia Neuhauser, Diane O'Dowd, Toto Olivera, Pavel Pevzner, Rebecca Richards-Kortum, Jasper Rine, Robert Sah, Scott Strobel, Graham Walker, David Walt, Isiah Warner, Sue Wessler, Huntington Willard, and Richard Zare 


\section{Science}

\section{Competencies: A Cure for Pre-Med Curriculum}

Winston A. Anderson, Richard M. Amasino, Manuel Ares Jr., Utpal Banerjee, Bonnie Bartel, Victor G. Corces, Catherine L.

Drennan, Sarah C. R. Elgin, Irving R. Epstein, Ellen Fanning, Louis J. Guillette Jr., Jo Handelsman, Graham F. Hatfull, Ronald Raymond Hoy, Darcy Kelley, Leslie A. Leinwand, Richard Losick, Yi Lu, David G. Lynn, Claudia Neuhauser, Diane K. O'Dowd, Toto Olivera, Pavel Pevzner, Rebecca R. Richards-Kortum, Jasper Rine, Robert L. Sah, Scott A. Strobel, Graham C. Walker, David R. Walt, Isiah M. Warner, Sue Wessler, Huntington F. Willard and Richard N. Zare

Science 334 (6057), 760-761.

DOI: $10.1126 /$ science.334.6057.760-b

ARTICLE TOOLS

http://science.sciencemag.org/content/334/6057/760.2

RELATED

http://science.sciencemag.org/content/sci/335/6074/1301.1.full

PERMISSIONS

http://www.sciencemag.org/help/reprints-and-permissions

Use of this article is subject to the Terms of Service

Science (print ISSN 0036-8075; online ISSN 1095-9203) is published by the American Association for the Advancement of Science, 1200 New York Avenue NW, Washington, DC 20005. The title Science is a registered trademark of AAAS.

Copyright $@$ 2011, American Association for the Advancement of Science 